\title{
Comparative proteomics analysis provides new insights into the haustorium development of Taxillus chinensis (DC.) Danser
}

\section{Limei Pan}

Garden of Medicinal Plant

Lingyun Wan

Garden of Medicinal Plant

Lisha Song

Garden of Medicinal Plant

Lili He

Garden of Medicinal Plant

Ni Jiang

Garden of Medicinal Plant

Hairong Long

Garden of Medicinal Plant

Juan Huo

Garden of Medicinal Plant

Xiaowen Ji

Garden of Medicinal Plant

Fengyun $\mathrm{Hu}$

Garden of Medicinal Plant

Jine Fu

Garden of Medicinal Plant

Shugen Wei ( $\nabla$ weishugen2@163.com )

Garden of Medicinal Plant

\section{Research Article}

Keywords: proteomics, T. chinensis, haustorium, phytohormone

Posted Date: November 30th, 2021

DOI: https://doi.org/10.21203/rs.3.rs-1033805/v1 
License: (c) (i) This work is licensed under a Creative Commons Attribution 4.0 International License. Read Full License 


\section{Abstract \\ Background}

Loranthus (Taxillus chinensis) is an important medicinal and parasitic plant that attacks other plants for living. To reveal the mechanisms of haustorium development, we employed an iTRAQ proteomics-based approach to identify differentially abundant proteins (DAPs) of fresh seeds (CK), baby (FB), and adult haustoria (FD).

\section{Results}

A total of 563 and 785 DAPs were successfully quantified in the early/later developmental stage, respectively. Pathway enrichment analysis indicated that the DAPs mainly associated with metabolic pathways, ribosome, phenylpropanoid biosynthesis and photosynthesis. In the meantime, DAPs associated with phytohormone signaling pathway changed markedly. Furthermore, we evaluated the contents change of phytohormone during the haustoria development. These results indicated that phytohormone is very important for haustorium development. qRT-PCR validation showed that the mRNA expression levels were consistent with the protein variation, suggesting that our result were reliable.

\section{Conclusions}

To the best of our knowledge, this is the first haustoria proteomes of loranthus, and our findings will improve our understanding of the molecular mechanism of haustoria development.

\section{Introduction}

The Taxillus chinensis (DC.) Danser is a member of the family Loranthaceae, which consists of approximately 73 genera and 900 species, which generally consists of aerial hemiparasitic plants (1). $T$. chinense is widespread distributed in the southern and southwestern areas of China. T. chinense is also used in Chinese traditional for many years, mainly because its stems and leaves can be used for the treatment of stroke, rheumatoid arthralgia, threatened abortion, angina pectoris, and hypertension (2). Mulberry, camellia, plum, peach, litchi, carambola, longan, and other economically important plant species can were parasitized by $T$. chinensis, which is semi-parasitic botanical medicinal materials (3). $T$. chinensis absorb nutrient compounds and water from the host plants via a unique multicellular organ termed the haustorium that aids to host attachment and invasion, although they provide their own carbohydrates through photosynthesis $(1,4)$.

In general, the initiation of haustorium development in most parasitic plants is triggered by host derived chemical signals terms haustorium-inducing factors (HIFs) after seed germination. Phenolic acids, quinones, flavonoids, and 2,6-dimethoxy-p-benzoquinone (DMBQ) that can be act as HIFs have been well 
documented (5). However, the exhaustive mechanisms about the how these compounds are released and then triggered haustorium development are still not well studied and need further research. Besides, some genes have been identified that may be involved in these processes. For example, the TVQR1 gene encoding quinone oxidoreductase is needed to trigger the development of the haustorium of the facultative parasite Triphysaria versicolor $(6,7)$. One possible reason for inducing the development of the haustor is that this enzyme converts quinone to semiquinone, which acts as an intermediate product during the redox cycle. Semiquinone itself or the redox cycle may be involved in haustorium induction signal transduction pathway $(6,8)$. The current model proposes that the phenolic acid derived from the degradation of the host cell wall is oxidized by ROS and oxidase to generate the haustor-inducing factor quinone $(9,10)$. In addition, it has been recently demonstrated that the accumulation of auxin and ethylene can act as early events in haustorium development of the hemiparasitic plant $T$. versicolor (11). Some research have observed that zeatin (Z), zeatin nucleotide (ZN), zeatin riboside (ZR), abscisic acid (ABA), and cytokinins (CKs) were significantly deposited in the haustoria of the Rhinanthus-Hordeum vulgare association $(12,13)$. Santalum album is a kind of invasive root hemiparasite. After being pretreated with 2 8mM GA3 for $12 \mathrm{~h}$, the seeds of Santalum album can germinate in vitro on Murashige and Skoog medium or in sand without HIFs trigger within one month $(14,15,16)$. All these results suggest that the plant hormones may play a crucial role in controlling haustorium development.

With the rapid development in mass spectrometry (MS), next-generation sequencing, and microarray technologies, the researchers has enabled relatively high-throughput analyses of transcriptomes, proteomes, and metabolomes and obtained large-scale snapshot information on transcripts, proteins, and metabolites. The changes in the transcriptome of haustorial development in $T$. chinense has been investigated and has shown the possible mechanism of $T$. chinense haustorial development (2). But study of biological processes at the protein level provides more realistic information compared to the transcript level. This is because proteins have biological functions, and the post-translational process will lead to the production of different protein subtypes (17). However, few studies have performed proteomic analysis of haustorial development in $T$. chinense.

\section{Methods}

\section{Plant Material}

The fresh seeds were collected from Taxillus chinensis (DC.) Danser which parasitized in the mulberry trees that were planted in the experimental field of Guangxi Botanical Garden of Medicinal Plants in China. Then, the seeds were peeled, washed with sterile water, placed on a germination dish, and incubated under the controlled environment $\left(25^{\circ} \mathrm{C}, 80 \%\right.$ relative humidity, $10 \mathrm{h:}: 14 \mathrm{~h}$ light: dark, $\left.2000 \mathrm{Lx}\right)$, as previously described (2). The fresh seeds were collected as control (CK). The seeds with protruding seed-type radicle and tiny suction device were collected after ten days of incubation (FB). And twenty days later, seeds with the loranthus haustoria and true leaves were used for the experiments (FD). Then, the samples were immediately frozen in liquid nitrogen, and stored at $-80^{\circ} \mathrm{C}$ until use. 


\section{Protein extraction}

Protein was extracted as described in a previous study (18). Briefly, the seeds samples were frozen in liquid nitrogen and pulverized. The samples were mixed with 5 volumes of chilled acetone containing $10 \%(\mathrm{v} / \mathrm{v})$ trichloroacetic acid (TCA) and incubated at $-20^{\circ} \mathrm{C}$ overnight. After centrifugation at $6,000 \mathrm{~g}$ and $40 \mathrm{~min}$, the supernatant was discarded. The precipitant was washed three times with pre-cooling acetone and was air-dried. The pellet was dissolved in Lysis buffer (8 M urea,120 mM NaCl, $10 \mathrm{mM}$ EDTA, $1 \%$ Triton X100 and 1\% PMSF dissolved in $50 \mathrm{Mm}$ Tris-HCI PH 8.0). Finally, the supernatant was filtered through $0.22-\mathrm{mm}$ filters after centrifugation at $13,000 \mathrm{~g}$ for $10 \mathrm{~min}$ at $4{ }^{\circ} \mathrm{C}$. The concentration of the protein was quantified with the BCA Protein Assay Kit (Bio-Rad, USA) and the quality of the protein sample was measured by SDS-PAGE, respectively.

\section{Protein digestion and iTRAQ labeling}

For each sample, $100 \mu \mathrm{g}$ protein was used for digestion and iTRAQ labeling. Protein were reduced with 10 $\mathrm{mM} \mathrm{DTT}$ at $37^{\circ} \mathrm{C}$ for $60 \mathrm{~min}$ and then alkylated with $55 \mathrm{mM}$ iodoacetamide (IAM) at room temperature for $30 \mathrm{~min}$ in darkness. The urea concentration of protein sample was diluted less than $2 \mathrm{M}$ by adding $100 \mathrm{mM}$ TEAB. Then trypsin was added to the protein pool of each sample with the ratio of protein: trypsin $=50: 1$ (mass ratio) at $37^{\circ} \mathrm{C}$ overnight and 100: 1 for a second digestion for $4 \mathrm{~h}$. After trypsin digestion, peptide was desalted with the Strata X SPE column and vacuum-dried. Peptide was reconstituted in $20 \mu \mathrm{L} 500 \mathrm{mM}$ TEAB and labeled according to the manufacturer's protocol. Briefly, dissolutioned the peptide solution in $50 \mu \mathrm{L}$ isopropanol and added one unit of ITRAQ reagent, incubated for $2 \mathrm{~h}$ at room temperature, then pooled and dried by vacuum centrifugation.

\section{High Performance Liquid Chromatography (HPLC) Fractionation}

The peptide was reconstituted with HPLC solution A ( $2 \%$ ACN, PH 10.0) and fractionated into fractions by high PH reverse-phase HPLC using Waters Bridge Peptide BEHC18 (130 $, 3.5 \mu \mathrm{m}, 4.6 * 250 \mathrm{~mm})$. Briefly, peptides were first separated with a gradient of $2 \%$ to $98 \%$ acetonitrile $(\mathrm{pH} 10)$ into 72 fractions. The wavelength $250 \mathrm{~nm}$ is used for detection of peptides. Then, the peptides were combined into 18 fractions and dried by vacuum centrifugation. The peptide fractions were desalted using Ziptip C18(Millipore, Billerica, MA) according to manufacturer's instructions.

\section{Liquid chromatography coupled with tandem mass spectrometry (LC-MS/MS) analysis}

Then the experiment was performed by NanoLC 1000 LC-MS/MS using a Proxeon EASY-nLC 1000 coupled to Thermo Fisher Q Exactive. Resuspended the disgestion fractions using $0.1 \%$ formic acid and loaded onto a reversed-phase-column (Acclaim PepMap $® 100$ C18, $3 \mu \mathrm{m}, 100 \AA, 75 \mu \mathrm{m} \times 2 \mathrm{~cm}$ ) at a rate of 5 $\mu \mathrm{L} / \mathrm{min}$ in $100 \%$ solvent $\mathrm{A} \otimes 0.1 \mathrm{M}$ acetic acid in water $\$. Next, peptides eluted from the trap column were loaded onto a reversed-phase analytical column (Acclaim PepMap®RSLC C18, $2 \mu \mathrm{m}, 100 \AA, 50 \mu \mathrm{m} \times$ $15 \mathrm{~cm})$. The gradient was comprised of an increase from $15 \%$ to $35 \%$ solvent $B(0.1 \%$ FA in $98 \% A C N)$ over $45 \mathrm{~min}, 35 \%$ to $98 \%$ solvent B during $5 \mathrm{~min}$ and keep in $98 \%$ in $5 \mathrm{~min}$ at a constant flow rate of 300 
$\mathrm{nl} /$ min on an EASY-nLC1000 system. The eluent was sprayed via NSI source at the 2.0 kv electrospray voltage and then analyzed by tandem mass spectrometry (MS/MS) in Q Exactive. A data-depentent mode in the scan range of $350-2000 \mathrm{~m} / \mathrm{z}$ was carried out for the mass spectrometry analyses, and the survey scans were captured at a mass resolution of 17500 by the Orbitrap analyzer. In the linear ion trap, 15 of the most intense precursor ions were selected for subsequent decision tree-based ion trap HCD fragmentation at the normalized collision energy of $32 \%$ in the MS survey scan with 10.0 s dynamic exclusion.

\section{Raw data processing}

The raw data files were searched against the transcriptome database using Swquest software integration in Proteome Discoverer (version 1.3, Thermo Scientific). The search parameters used as follows: Carbamidomethylation (C) was set as fixed modifications; and oxidation (M), acetylation in N-Term were set as variable modification. Trypsin was chosen as enzyme and two missed cleavages were allowed. Mass tolerance of precursor ions was 20 ppm, and a fragment ion tolerance was $0.05 \mathrm{Da}$, resulting in $1 \%$ false discovery rate (FDR). Differentially abundant proteins (DAPs) were identified based on the following criteria: P-values smaller than 0.05 and a mean relative abundance $>1.2$ or $<0.83$.

\section{Bioinformatics analysis}

To determine the functional characterization of differentially abundant proteins (DAPs), proteins were mapped with Gene Ontology (GO) annotation base on UniProt-GOA database (www.http://www.ebi.ac.uk/GOA) (19). All proteins were grouped into three major categories: biological processes, cellular components, and molecular functions. The metabolic pathway analyses of DAPs were based on the Kyoto Encyclopedia of Genes and Genomes (KEGG) database (http://www.genome.jp/kegg/) (20).

\section{Phytohormone analysis}

Phytohormone indole-3-acetic acid (IAA), gibberellin (GA), and abscisic acid (ABA) were quantified based on a liquid chromatography and mass spectrometry system (LC-MS, 1120-6460, Agilent, USA). Phytohormone extraction was performed from loranthus seeds based on the previous method (21). The phytohormone were separated by C18 column (Hypersil Gold, $100 \mathrm{~mm} \times 2.1 \mathrm{~mm}, 1.9 \mu \mathrm{m}$, Thermo Fisher Scientific) at flow rate of $0.3 \mathrm{~mL} / \mathrm{min}$ with a $17 \mathrm{~min}$ gradient elution. For Multiple Reaction Monitoring (MRM), the phytohormone were analyzed with negative mode by ESI ion source.

\section{Quantitative real-time PCR analysis}

Total RNA was extracted from each sample using TRIzol reagent (Invitrogen) according to the manufacturer's protocol. FastQuant RT Kit (with gDNase, Tiangen) was used for DNA remove and cDNA synthesis. Specific primer pairs of randomly selected 8 genes for quantitative real-time PCR (qRT-PCR) were designed using Primer Premier 5.0 software and sequences are listed in Table S1. actin was used as 
reference gene (2). The procedure of qRT-PCR experiment was same as our previous study (22). $2^{-\Delta \Delta C t}$ method was used to evaluate the expression levels of transcripts in each sample (23). Each transcript was performed with three biological replicates and three technical replicates.

\section{Result}

\section{Differential abundant proteins (DAPs) analysis}

To investigate the potential mechanisms involved in the haustorial developmental process in $T$. chinense, an integrated approach involving LC-MS/MS and iTRAQ labeling was applied to analyze the proteomic changes. A total of 563 proteins were identified as DAPs between FB treatment and control, of which 384 were identified as upregulated and 179 were downregulated proteins, respectively. A total of 785 proteins were identified as DAPs between FD treatment and control, of which 569 and 216 were identified as upregulated and downregulated proteins, respectively (Figure 1). All the DAPs were grouped based on their subcellular localizations. For the FB treatment, 11 subcellular components were identified, including 250 chloroplast-localized proteins (44.4\%), 162 cytosol- localized proteins $(28.77 \%), 80$ nuclear-localized proteins $(14.21 \%)$. For the FD treatment, 16 subcellular components were identified, including 325 chloroplast-localized proteins (41.4\%), 2433 cytosol- localized proteins (30.96\%), 106 nuclear-localized proteins (13.5\%) (Figure 1).

\section{Functional categorization analysis}

The biological functions of the DAPs could also be identified by their GO annotations. The DAPs were classified into the three main GO categories (cellular component, biological process, and molecular function). In the early developmental stage (FB), 1546 DAPs (some proteins have more than one GO annotation) were annotated with biological process, 653 DAPs with molecular functions, and 1885 DAPs with cellular component compared to control. In the later developmental stage (FD), 2126 DAPs were annotated with biological process, 945 DAPs with molecular functions, and 2665 DAPs with cellular component compared to control. In the biological process, the most enriched categories were cellular process and metabolic process. In the molecular function, the most enrichment occurred in relation to binding and catalytic activity. In the cellular component, the most enrichment occurred in relation to cell and cell part (Figure 2).

\section{Metabolism pathway analysis}

To further understand molecular mechanisms potentially associated with haustorial developmental in $T$. chinense, DAPs were subjected to the KEGG pathway database. During the early developmental stage (FB), the proteins with increased abundance were mainly involved in pathways related to metabolic pathways (108 DAPs), photosynthesis (20 DAPs), and carbon fixation in photosynthetic organisms (12 DAPs). the proteins with increased abundance were involved in only three pathways, namely ribosome (52 DAPs), systemic lupus erythematosus (3 DAPs), and flavonoid biosynthesis (3 DAPs). During the later developmental stage (FD), the proteins with increased abundance were mainly involved in pathways 
related to metabolic pathways (158 DAPs), photosynthesis (23 DAPs), glyoxylate and dicarboxylate metabolism (15 DAPs), and phenylpropanoid biosynthesis (14 DAPs). In addition, the proteins whose abundance decreased were related to also only three pathways, which are ribosome (67 DAPs), systemic lupus erythematosus (4 DAPs), and alcoholism (4 DAPs) (Figure 3).

\section{DAPs involved in phytohormone signaling pathway}

Based on the proteomic analysis, the levels of 19 proteins associated with phytohormone signaling pathway were altered, including auxin-binding protein, mitogen-activated protein kinase, $A B C$ transporter protein, and gibberellin-regulated protein and so on (Table S2). We found that the majority of DAPs related to auxin signaling pathways were significantly increased during the haustorial developmental in $T$. chinense, with a highest ratio of 2.34 for enhancer of mRNA-decapping protein 4-like (Table S2). Only glutaredoxin was significantly down regulated with a ratio of 0.47 and 0.37 , respectively. Furthermore, all four proteins associated with abscisic acid signaling pathway were significantly up regulated with a ratio that ranged from 1.57 to 2.58 . In addition to, three DAPs associated with gibberellin signaling pathway abundance was marked down regulated, with a lowest ratio of 0.09 being observed for GAST-like protein after 10 days of haustorial developmental.

\section{Phytohormone concentrations}

To elucidate the correspondence between the level of hormone and abundance of proteins, the IAA, ABA, and GA contents were analyzed by LC-MS. The accumulation of IAA was slightly decreased after 10 days of haustorial developmental. However, content of IAA was significantly increased after 20 days of haustorial developmental compared to the $\mathrm{CK}$ and FB. In contrast, the levels of $\mathrm{ABA}$ gradually decreased with the haustorial developmental. Interestingly, GA3 contents was initially up regulated, but then down regulated, reaching its highest level at 10 days (Figure 4).

\section{Transcriptional expression analysis by qRT-PCR}

To confirm the correspondence between the transcript level of mRNA and abundance of protein, transcriptional analysis of 8 DAPs was analyzed by qRT-PCR. The results showed that expressions of these gene were similar to those the abundance of their corresponding protein. The results indicate that the method used to confirm DAPs in this experiment is feasible (Figure 5).

\section{Discussion}

Branches and leaves of $T$. chinensis are widely used in traditional Chinese medicines for treating rheumatism, hypertension, and obesity as well as for preventing miscarriages (24). The haustorium is an essential parasitic organ used by $T$. chinensis to penetrate host tissues. However, the molecular mechanism of $T$. chinensis haustorium developmental is still unclear. To gain insights on protein changes in the developmental reprogramming of haustorial formation in T. chinensis, a comparative proteomic approach based on ITRAQ strategy was undertaken to study detailed proteome changes during its 
development. A total of 563 and 785 DAPs were successfully identified in in the early/later developmental stage, respectively. These DAPs were functionally classified according to their roles and were enriched in metabolic pathways, ribosome, phenylpropanoid biosynthesis and phytohormone signaling pathway. Our results provide information for the further identification and functional study of proteins in $T$. chinensis.

Some studies have uncovered metabolic pathways have important function in haustorial development. Ichihashi et al proposed that the accumulation of very long chain fatty acid (VLCFAs) involved in the developmental reprogramming of Thesium chinense haustorial formation in natural environment (25). Besides, some metabolites have been demonstrated response to haustorial development. For example, lignin-related compounds induced haustoria in $P$. japonicum and $S$. hermonthica with different specificities. High concentrations of lignin polymers induced haustorium formation. Treatment with laccase, a lignin degradation enzyme, promoted haustorium formation at low concentrations (26). In our study, 15 up regulated DAPs involved in phenylpropanoid biosynthesis were identified. Phenylpropanoid metabolism is one of the most important metabolisms in plants, contributing to plant development (27). The biosynthetic pathways leading to lignin is known to be originated from the general phenylpropanoid pathway (28). Together with these results collectively imply that the accumulation of lignin, leading to the T. chinensis haustorium formation, was regulated key proteins belong to phenylpropanoid metabolism pathway.

Phytohormones, such as IAA, GAs, ABA, play crucial roles in regulating plant growth and development and responding to various stresses as previously reported $(29,30)$. Auxin was demonstrated that involved in the formation of cluster-roots (CR) and adventitious roots (AR) (31). Genes related to auxin singling pathway were significantly enriched in the parasitic plant $T$. chinense and $C$. australis. (32). In the meantime, genes involved in polar auxin transport were also enriched haustorial and prehaustorial stages compared to reference tissues, stems and seedlings of dodder. (33). Polar auxin transport promotes the formation of local auxin maxima and gradients within tissues and further results in patterns of cell division and differentiation in the root meristem (34). And the accumulation of auxin level was also observed in the haustoria initiation in sandalwood (15). Our result demonstrated that content of auxin was significantly increased after 20 days of haustorial developmental, which was consistent with the protein related to auxin abundance. GA3 was also considered as an important regulatory factor in haustorial development of parasitic plants, which may originated from effects of GA3 on xylem formation and elongation (35). Endogenous GA3 was involved in tracheary element differentiation was reported in Zinnia elegans xylogenic culture (36). In parasite-host associations, ABA is considered as a root-derived signaling molecule. In general, ABA levels in parasite roots were also higher than in the host root, which to keep stomata closed and the hydraulic conductivity of roots response to higher transpiration (12). However, our study suggested that the levels of ABA gradually decreased with the haustorial developmental. A more precise mechanism of the ABA function still need further investigation. In the meantime, we observed that the protein abundance related to ABA was significantly up regulated. We speculate these proteins may as negative regulator to control ABA biosynthesis. All these results imply that endogenous auxin, GAs, and ABA may function as an important regulatory factor during the haustorial developmental in T. chinense. 


\section{Conclusions}

In conclusion, we studied the protein and phytohormones profiles of the loranthus haustoria development. Some crucial proteins involved in haustorial developmental were successfully identified in this work. More importantly, the findings of this study will improve our understanding of parasitism and contribute to the breeding program of loranthus.

\section{Abbreviations}

DAPs: differentially abundant proteins; CK: fresh seeds; FB: baby haustoria; FD: adult haustoria; qRT-PCR: quantitative real-time PCR; DMBQ: 2,6-dimethoxy-p-benzoquinone; HIF: haustorium-inducing factor; CKs: cytokinins; Z: zeatin; ZR: zeatin riboside; ZN: zeatin nucleotide; ABA: abscisic acid; GA: gibberellin; TCA: trichloroacetic acid; HPLC: high performance liquid chromatography; LC-MS/MS: liquid chromatography coupled with tandem mass spectrometry; FDR: false discovery rate; GO: Gene Ontology; KEGG: Kyoto Encyclopedia of Genes and Genomes; MRM: multiple reaction monitoring; VLCFAs: very long chain fatty acid; CR: cluster-roots; AR: adventitious roots.

\section{Declarations}

\section{Ethics approval and consent to participate}

Not applicable.

\section{Consent for publication}

Not applicable.

\section{Availability of data and material}

The datasets used and analyzed during the current study could be available from the corresponding author on reasonable request.

\section{Competing interests}

The authors declare that they have no competing interests.

\section{Funding}

This work was supported by the National Natural Science Foundation of China $(81860672,81960695$, and 82173933), the Guangxi Natural Science Foundation, China (2017GXNSFDA198026, 2018GXNSFAA281089, and 2021 GXNSFBA075037), the Guangxi Botanical Garden of Medicinal Plants Research and Innovation Team Building Project (GYCH2019008) and the scientific research funding project of Guangxi Botanical Garden of Medicinal Plants (GYJ202012). 


\section{Authors' Contributions}

SW, JF, and LP conceived and designed the experiments. LP, WL, and SJ performed the experiments. LP, $\mathrm{HL}, \mathrm{JN}, \mathrm{LH}$, and $\mathrm{HJ}$ analyzed the data. LP, JX, and HF wrote the manuscript. LP revised the manuscript. All the authors have read and approved the final version of manuscript.

\section{Acknowledgments}

Not applicable.

\section{References}

1. Moghadamtousi SZ, Kamarudin MNA, Chan CK, Goh BH, Kadir HA. (2014). Phytochemistry and biology of Loranthus parasiticus Merr, a commonly used herbal medicine. American Journal of Chinese Medicine. 42:23-35. Doi: 10.1142/S0192415X14500025.

2. Wei SG, Wan LY, He LL, Wei Y, Long HR, Ji XW, Fu JE, Pan LM. (2020). De novo transcriptome reveals gene changes in the development of the endosperm chalazal haustorium in Taxillus chinensis (DC.). Danser. Biomed Research International. 7871918. Doi: 10.1155/2020/7871918.

3. Liu RY, Su BW, Huang FY, Ru M, Zhang H, Qin ZM, Li YH, Zhu KX. (2019). Identification and analysis of cardiac glycosides in Loranthaceae parasites Taxillus chinensis (DC.) Danser and Scurrula parasitica Linn. and their host Nerium indicum Mill. Journal of Pharmaceutical and Biomedical Analysis. 174: 450-459. Doi: 10.1016/j.jpba.2019.05.071.

4. Yoshida S, Cui SK, Ichihashi Y, Shirasu K. (2016). The haustorium, a specialized invasive organ in parasitic plants. Annual Review of Plant Biology. 67: 643-667. Doi: 10.1146/annurev-arplant043015-111702.

5. Yoneyama K, Xie XN, Sekimoto H, Takeuchi Y, Ogasawara S, Akiyama K, Hayashi, H, Yoneyama K. (2008). Strigolactones, host recognition signals for root parasitic plants and arbuscular mycorrhizal fungi, from Fabaceae plants. New Phytologist. 179: 484-494. Doi: 10.1111/j.14698137.2008.02462.x.

6. Bandaranayake PCG, Filappova T, Tomilov A, Tomilova NB, Jamison-McClung D, Ngo Q, Inoue K, Yoder JI. (2010). A single-electron reducing quinone oxidoreductase is necessary to induce haustorium development in the root parasitic plant triphysaria. Plant Cell. 22: 1404-1419. Doi: 10.1105/tpc.110.074831.

7. Bandaranayake PCG, Tomilov A, Tomilova NB, Ngo Q, Wickett N, dePamphilis CW, Yoder JI. (2012). The TvPirin gene is necessary for haustorium development in the parasitic plant Triphysaria versicolor. Plant Physiology. 158: 1046-1053. Doi: 10.1104/pp.111.186858.

8. Kim DJ, Kocz R, Boone L, Keyes WJ, Lynn DG. (1998). On becoming a parasite: evaluating the role of wall oxidases in parasitic plant development. Chemistry Biology. 5: 103-117. Doi: 10.1016/S10745521(98)90144-2. 
9. Westwood JH, Yoder $\mathrm{JI}$, TimkoMP, dePamphilis CW. (2010). The evolution of parasitism in plants. Trends in Plant Science. 15: 227-235. Doi: 10.1016/j.tplants.2010.01.004.

10. Cui S, Wakatake T, Hashimoto K, Saucet SB, Toyooka K, Yoshida S, Shirasu K. (2016). Haustorial hairs are specialized root hairs that support parasitism in the facultative parasitic plant Phtheirospermum japonicum. Plant Physiology. 170:1492-1503. Doi: 10.1104/pp.15.01786.

11. Tomilov AA, Tomilova NB, Abdallah I, Yoder JI. (2005). Localized hormone fluxes and early haustorium development in the hemiparasitic plant Triphysaria versicolor. Plant Physiology. 138: 1469-1480. Doi: 10.1104/pp.104.057836.

12. Jiang F, Jecchke WD, Hartung W. (2004). Abscisic acid (ABA) flows from Hordeum vulgare to the hemiparasite Rhinanthus minor and the influence of infection on host and parasite abscisic acid relations. Journal of Experimental Botany. 55: 2323-2329. Doi: 10.1093/jxb/erh240.

13. Jiang F, Veselova S, Veselov D, Kudoyarova G, Jeschke WD, Hartung W. (2005). Cytokinin flows from Hordeum vulgare to the hemiparasite Rhinanthus minor and the influence of infection on host and parasite cytokinins relations. Functional Plant Biology. 32: 619-629. Doi: 10.1071/FP04168.

14. Nikam TD, Barmukh RB. (2009). GA3 enhances in vitro seed germination in Santalum album. Seed Science and Technology. 37: 276-280. Doi: https://doi.org/10.15258/sst.2009.37.2.02.

15. Zhang XH, da Silva JAT, Duan Jun, Deng RF, Xu XL, Ma GH. (2012). Endogenous hormone levels and anatomical characters of haustoria in Santalum album L. seedlings before and after attachment to the host. Journal of Physiology. 169: 859-866. Doi: 10.1016/j.jplph.2012.02.010.

16. Barrett DR, Fox JED. (1997). Santalum album: kernel composition, morphological and nutrient characteristics of pre-parasitic seedlings under various nutrient regimes. Annals of Botany. 79: 5966. Doi: $10.1006 /$ anbo.1996.0303.

17. Hegde PS, White IR, Debouck, C. (2003). Interplay of transcriptomics and proteomics. Current Opinion Biotechnology. 14: 647-651. Doi: 10.1016/j.copbio.2003.10.006.

18. Isaacson T, Damasceno CMB, Saravanan RS, He Y, Catala C, Saladie M, Rose JKC. (2006) Sample extraction techniques for enhanced proteomic analysis of plant tissues. Nature Protocols. 1:769774. Doi:10.1038/nprot.2006.102.

19. Camon E, Magrane M, Barrell D, Lee V, Dimmer E, Maslen J, Binns D, Harte N, Lopez N, Apweiler R. (2004). The gene ontology annotation (GOA) database: sharing knowledge in Uniprot with gene ontology. Nucleic Acids Research. 32: 262-266. Doi: 10.1093/nar/gkh021.

20. Kanehisa M, Goto S. (2000). KEGG: Kyoto Encyclopedia of Genes and Genomes. Nucleic Acids Research. 28:27-30. Doi: 10.1093/nar/28.1.27.

21. Pan LM, Wan LY, He LL, Song LS, Long HR, Ji XW, Jiang N, Huo J, Wei SG, Fu JE. (2021). Comparative proteomic analysis of parasitic loranthus seeds exposed to dehydration stress. Plant Biotechnology Reports. 15: 95-108 Doi: 10.1007/s11816-020-00651-4.

22. Wei SG, Ma XJ, Pan LM, Miao JH, Fu JN, Bai LH, Zhang ZL, Guan YH, Mo CM, Huang H, Chen MS. (2017). Transcriptome analysis of Taxillusi chinensis (DC.) danser seeds in response to water loss. Plos One. 12:e0169177. Doi: 10.1371/journal.pone.0169177. 
23. Pfaffl MW. (2001) A new mathematical model for relative quantification in real-time RT-PCR. Nucleic Acids Research. 29: e45. Doi: 10.1093/nar/29.9.e45.

24. Ding B, Dai Y, Hou YL, Wu XM, Chen X, Yao XS. (2013). Four new hemiterpenoid derivatives from Taxillus chinensis. Fitoterapia. 86:1-5. Doi: 10.1016/j.fitote.2013.01.017

25. Ichihashi Y, Kusano M, Kobayashi M, Suetsugu K, Yoshida S, Wakatate T, Kumaishi K, Shibata A, Saito K, Shirasu K. (2018). Transcriptomic and metabolomic reprogramming from roots to haustoria in the parasitic plant, Thesium chinense. Plant and Cell Physiology. 59: 729-737. Doi:

10.1093/pcp/pcx200.

26. Cui S, Wada S, Tobimatsu Y, Takeda Y, Saucet SB, Takano T, Umezawa T, Shirasu K, Yoshida S. (2018). Host lignin composition affects haustorium induction in the parasitic plants Phtheirospermum japonicum and Striga hermonthica. New Phytologist. 218: 710-723. Doi: 10.1111/nph.15033.

27. Dong NG, Lin HX. (2021). Contribution of phenylpropanoid metabolism to plant development and plant-environment interactions. Journal of Integrative Biology. 63: 180-209. Doi:

10.1111/jipb.13054.

28. Zhang S, Yang J, Li HQ, Chiang VCL, Fu YJ. (2021). Cooperative regulation of flavonoid and lignin biosynthesis in plants. Critical Reviews in Plant Sciences. 40: 109-126. Doi: 10.1080/07352689.2021.1898083.

29. Suzaki T, Ito M, Kawaguchi M. (2013). Genetic basis of cytokinin and auxin functions during root nodule development. Frontiers in Plant Science. 4: 1-6. Doi: 10.3389/fpls.2013.00042.

30. Erb M, Meldau S, Howe GA. (2012). Role of phytohormones in insect-specific plant reactions. Trends in Plant Science. 17: 250-259. Doi: 10.1016/j.tplants.2012.01.003.

31. Pacurar DI, Perrone I, Bellini C. (2014). Auxin is a central player in the hormone cross-talks that control adventitious rooting. Physiologia Plantarum. 151: 83-96. Doi: 10.1111/ppl.12171.

32. Sun GL, Xu YX, Liu H, Sun T, Zhang JX, Hettenhausen C, Shen GJ, Qi JF, Qin Y, Li J, Wang L, Chang W, Guo ZH, Baldwin IT, Wu JQ. (2018). Large-scale gene losses underlie the genome evolution of parasitic plant Cuscuta australis. Nature Communications. 9: 2683. Doi: 10.1038/s41467-01804721-8.

33. Ranjan A, Ichihashi Y, Farhi M, Zumstein K, Townsley B, David-Schwartz R, Sinha NR. (2014). De novo assembly and characterization of the transcriptome of the parasitic weed dodder identifies genes associated with plant parasitism. Plant Physiology. 166: 1186-1199. Doi: 10.1104/pp.113.234864.

34. Frim J. (2003). Auxin transport - shaping the plant. Current Opinion in Plant Biology. 6:7-12. Doi: $10.1016 /$ S1369526602000031.

35. Eriksson ME, Israelsson M, Olsson O, Moritz T. (2000). Increased gibberellin biosynthesis in transgenic trees promotes growth, biomass production and xylem fiber length. Nature Biotechnology. 18:784-8. Doi: $10.1038 / 77355$

36. Tokunaga N, Uchimura N, Sato Y. (2006). Involvement of gibberellin in tracheary element differentiation and lignification in Zinnia elegans xylogenic culture. Protoplasma. 228: 179-187. Doi: 


\section{Figures}

a

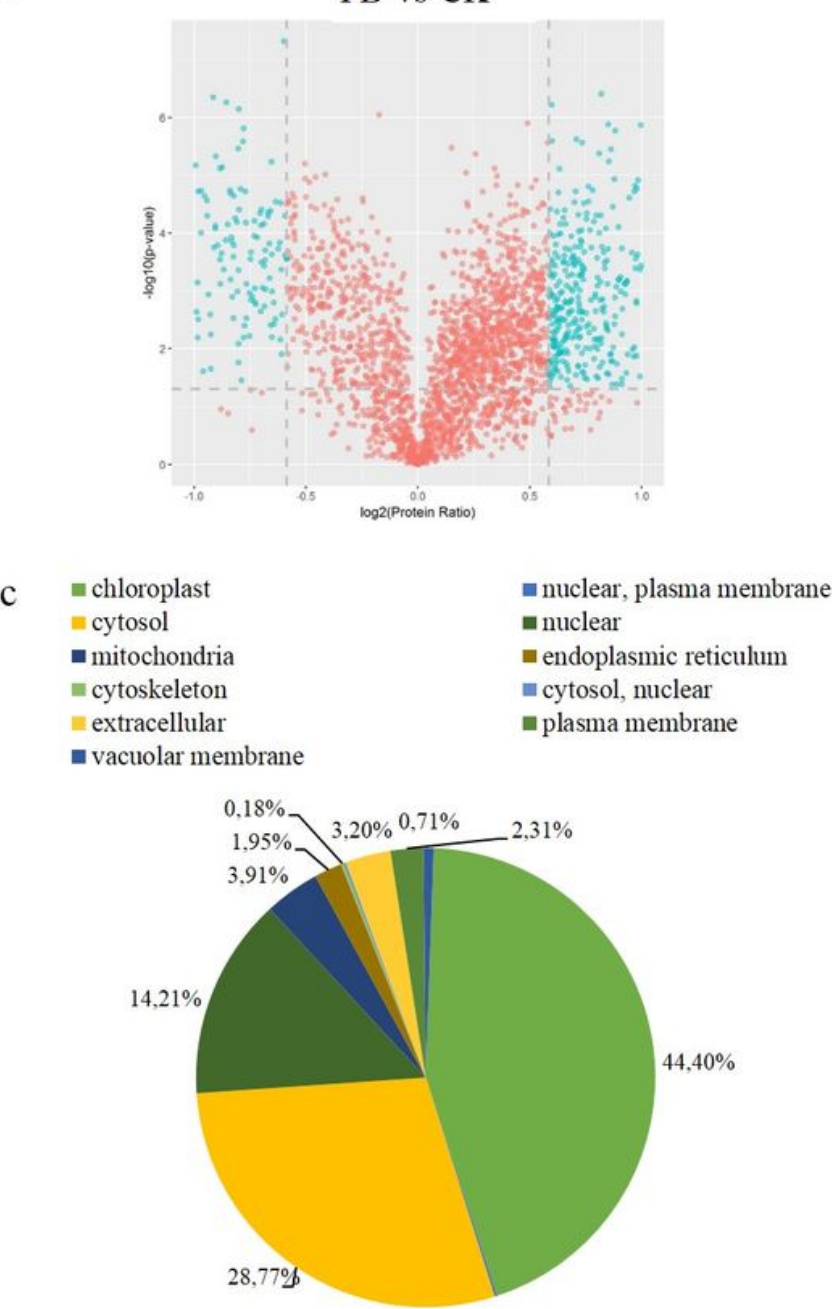

b

FD-vs-CK

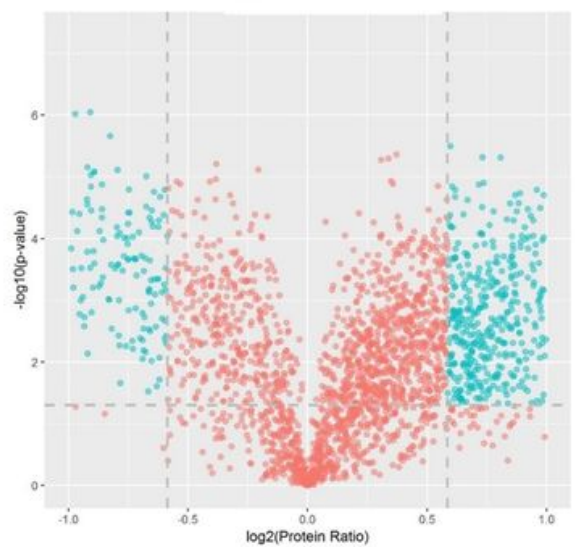

d

\begin{tabular}{|c|c|}
\hline $\begin{array}{l}\text { - chloroplast } \\
\text { = nuclear, plasma membrane } \\
\text { - nuclear } \\
\text { = endoplasmic reticulum }\end{array}$ & $\begin{array}{l}\text { a Golgi apparatu } \\
\text { a cytosol } \\
\text { mitochondria } \\
\text { m peroxisome }\end{array}$ \\
\hline
\end{tabular}

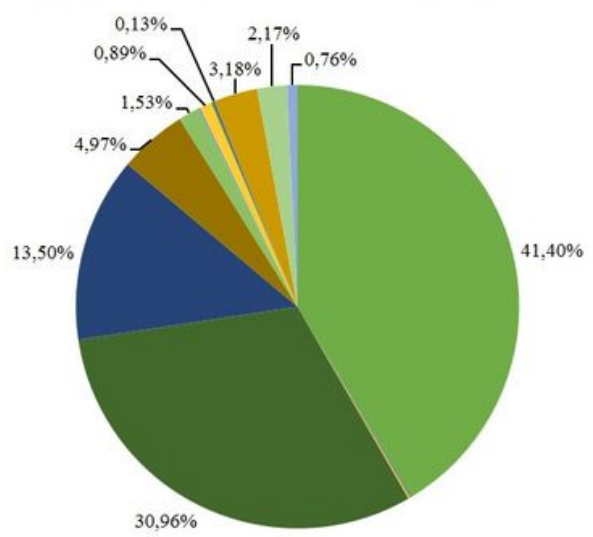

Figure 1

Identification of differentially abundant proteins (DAPs) between FB vs CK (a) and FD vs CK (b). Subcellular locations of differentially abundant proteins (DAPs) between FB vs CK (c) and FD vs CK (d). 
a

Enriched GO Terms

(FB vs CK)

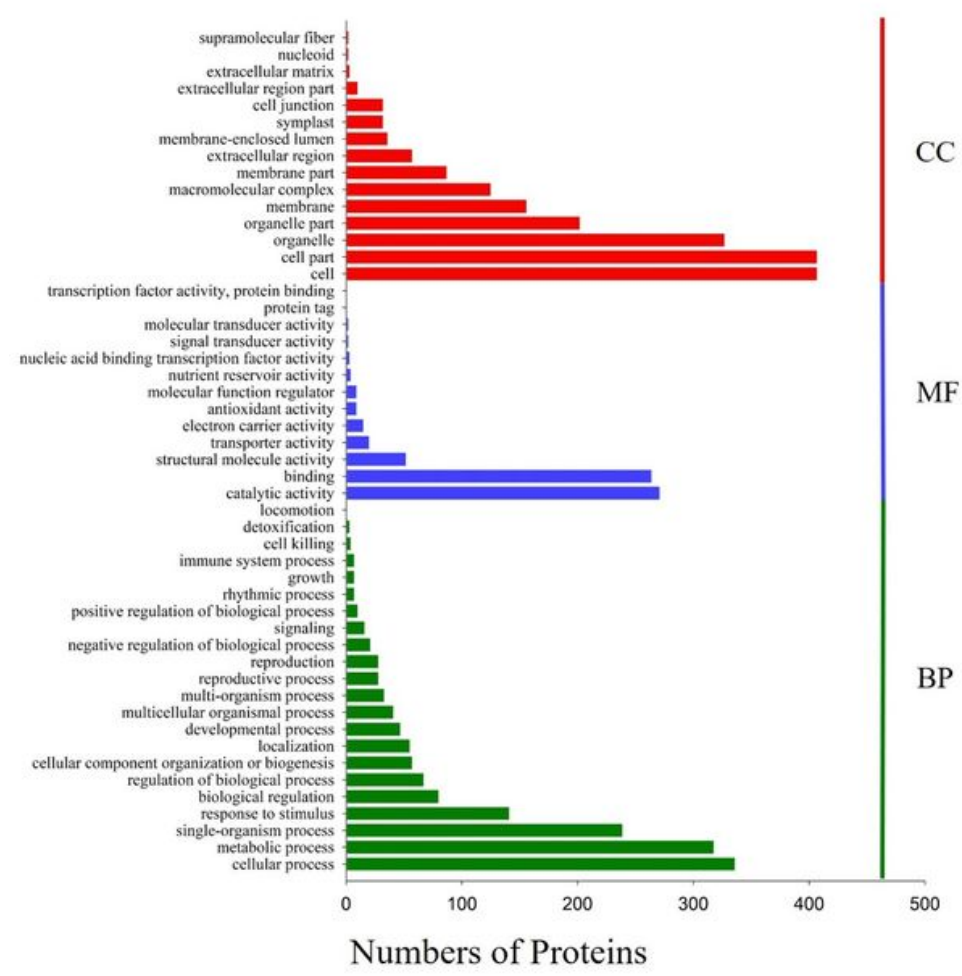

$\mathrm{CC}$ b Enriched GO Terms

(FB vs $\mathrm{CK}$ )

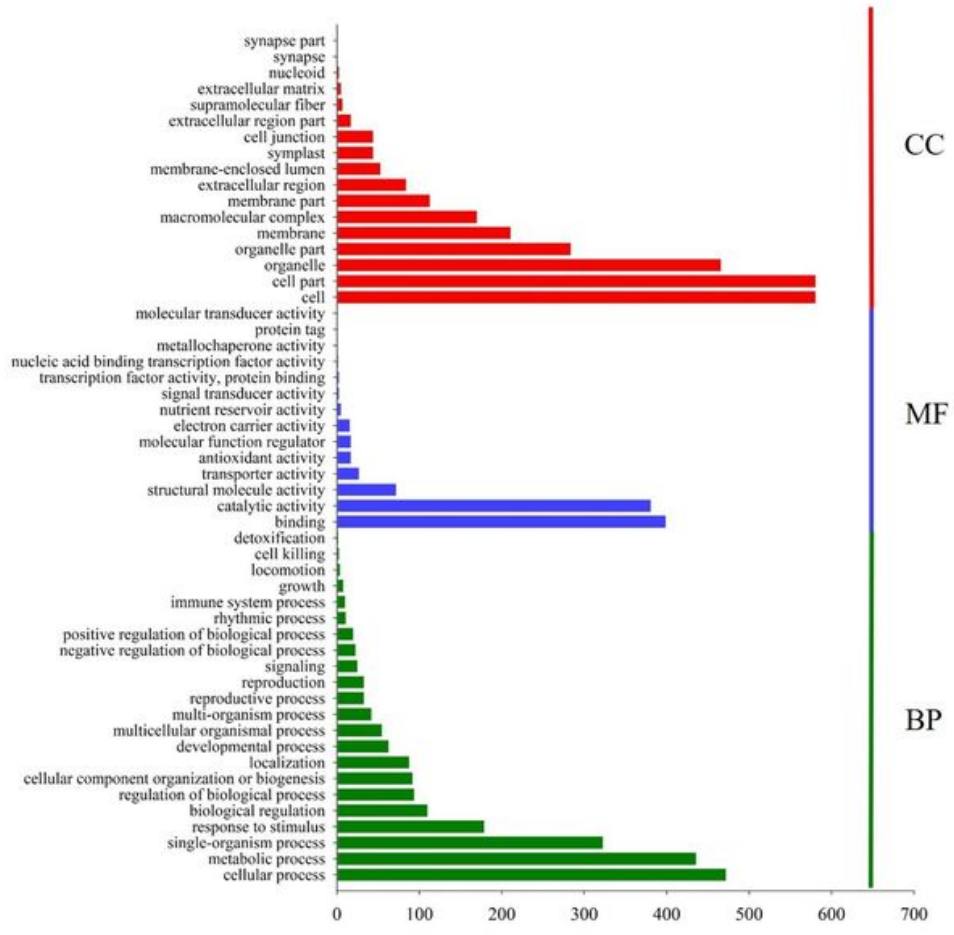

Numbers of Proteins

Figure 2

Gene Ontology (GO) analysis of differentially abundant proteins (DAPs) between FB vs CK (a) and FD vs CK (b). And the proteins were annotated by biological process, cellular component, and molecular function. 
a

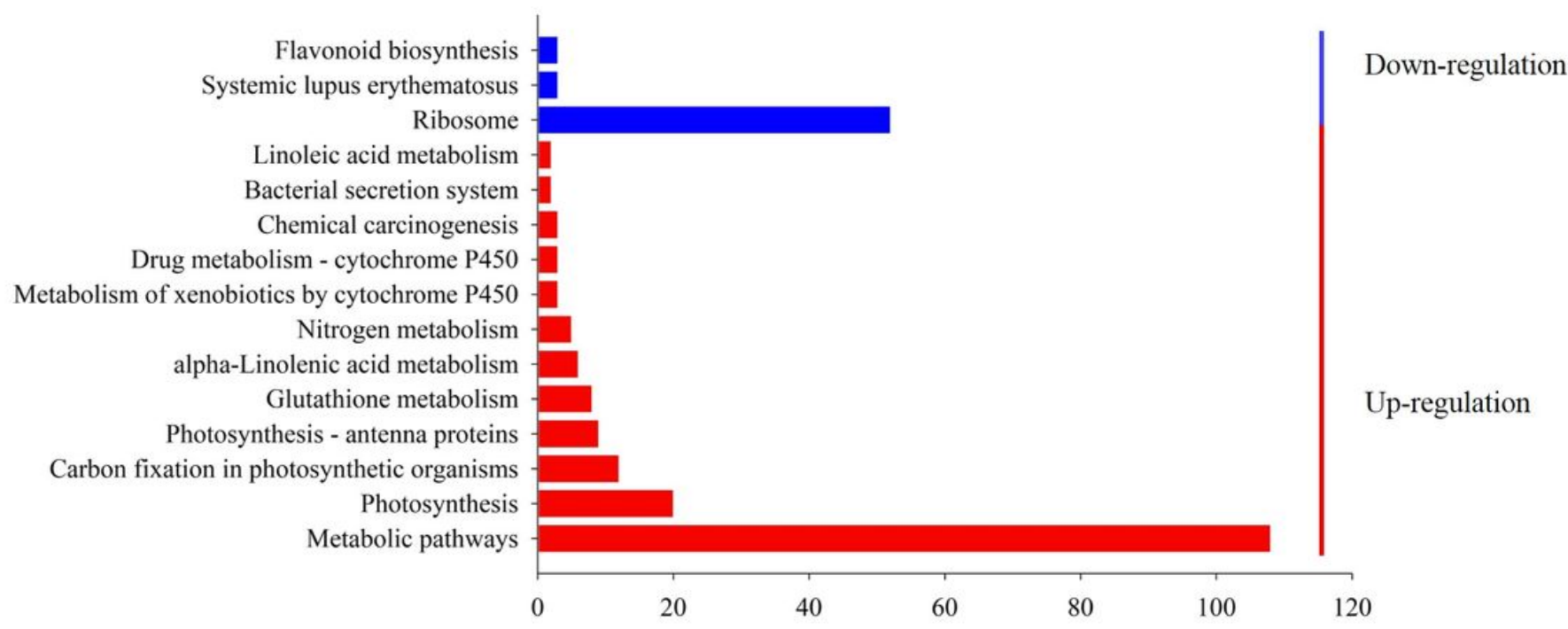

Numbers of Proteins

b

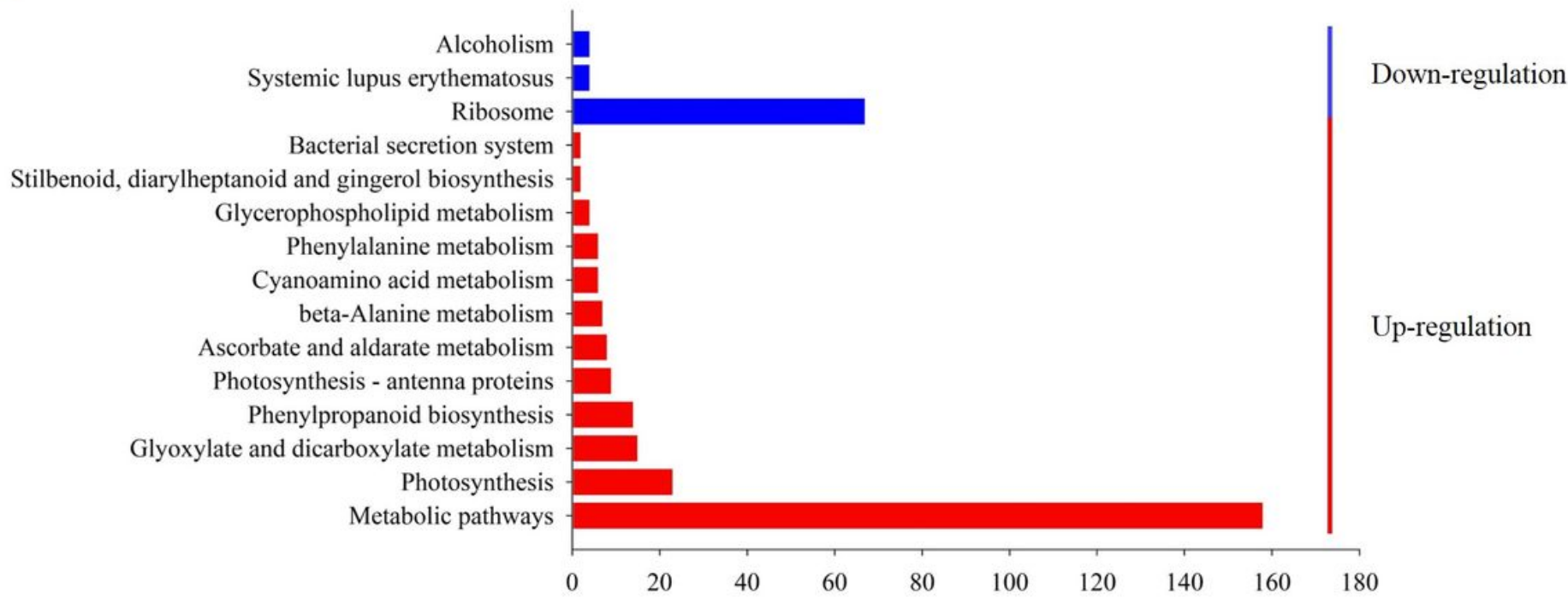

Numbers of Proteins

Figure 3

Kyoto Encyclopedia of Genes and Genomes (KEGG) analysis of differentially abundant proteins (DAPs) between FB vs CK (a) and FD vs CK (b). 


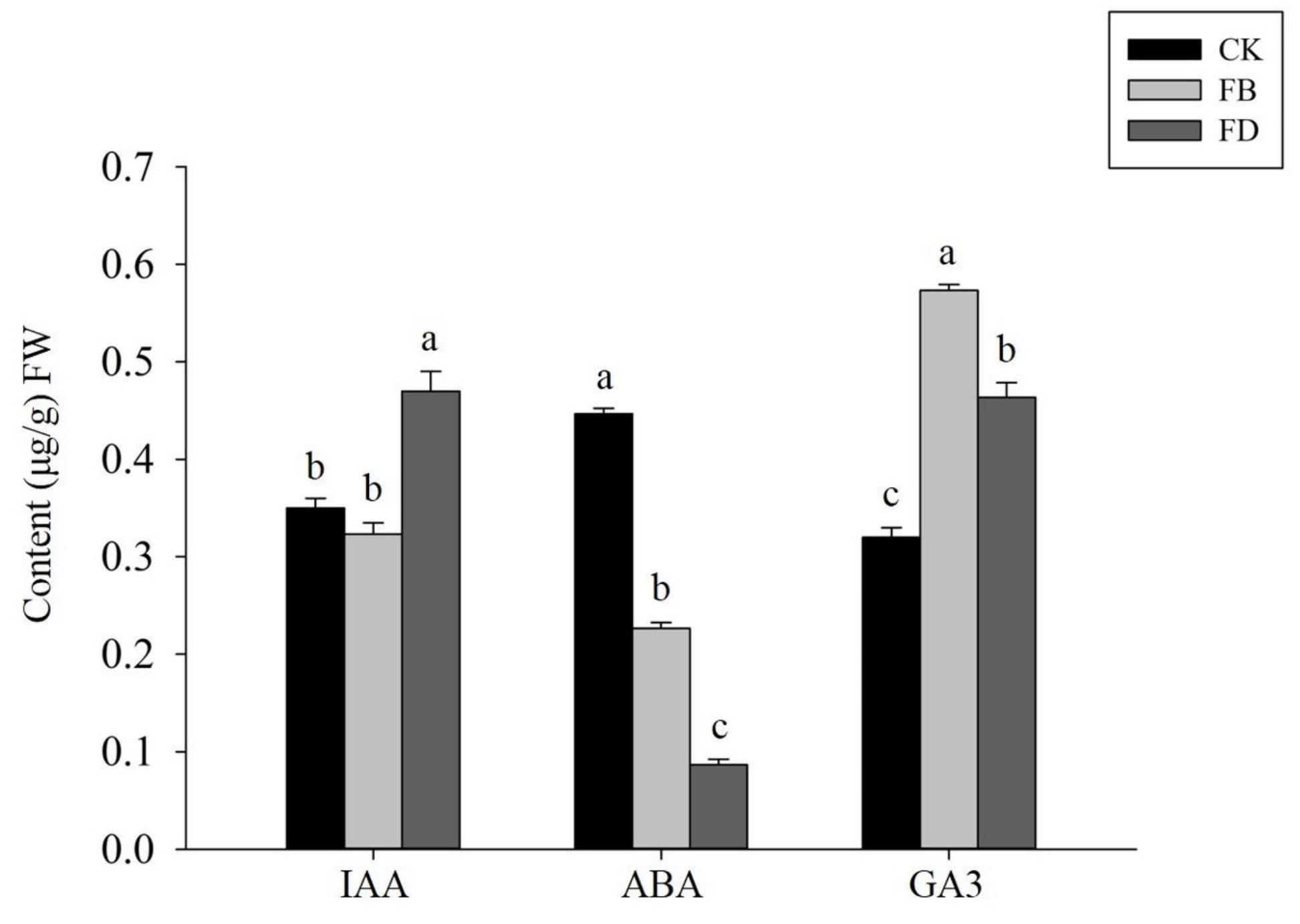

Figure 4

Average phytohormone concentrations of IAA, ABA, and GA3 in FB, FD, and control plants. Different letters above the bars indicate significant differences, $p<0.05$. 


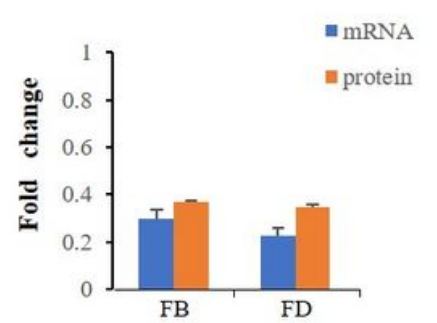

Unigene8035_All

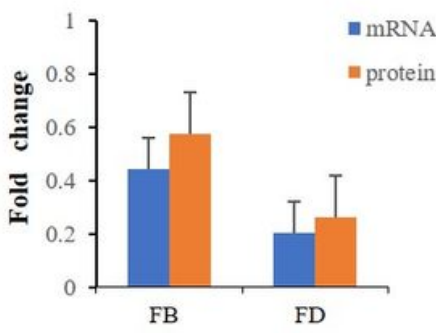

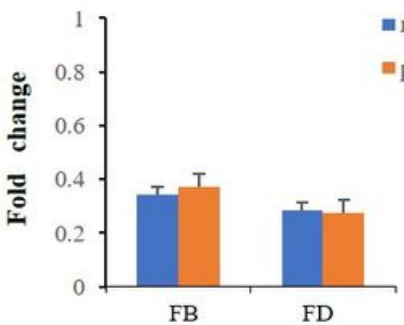

CL9588.Contig3_All

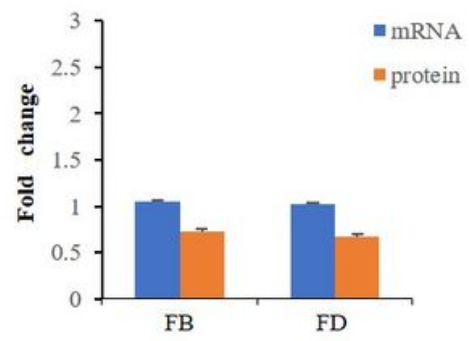

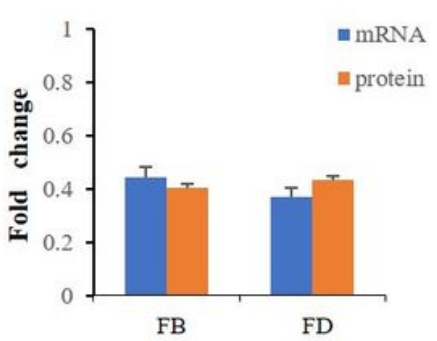

Unigene23479_All

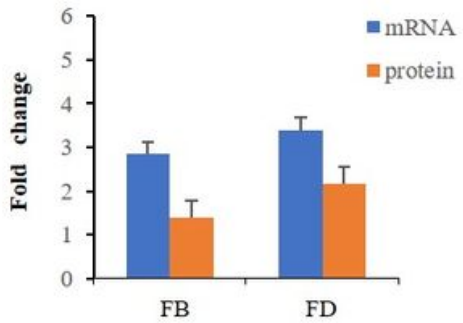

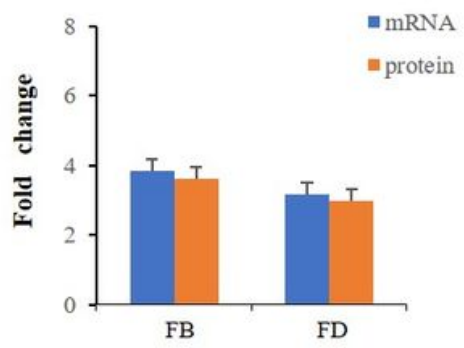

Unigene2669 All

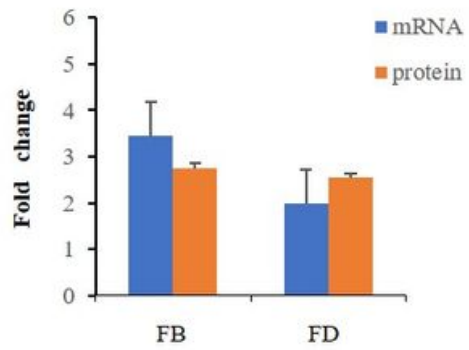

Figure 5

Analysis of the corresponding transcript levels of proteins by qRT-PCR.

\section{Supplementary Files}

This is a list of supplementary files associated with this preprint. Click to download.

- TableS1.xlsx

- TableS2.xlsx 